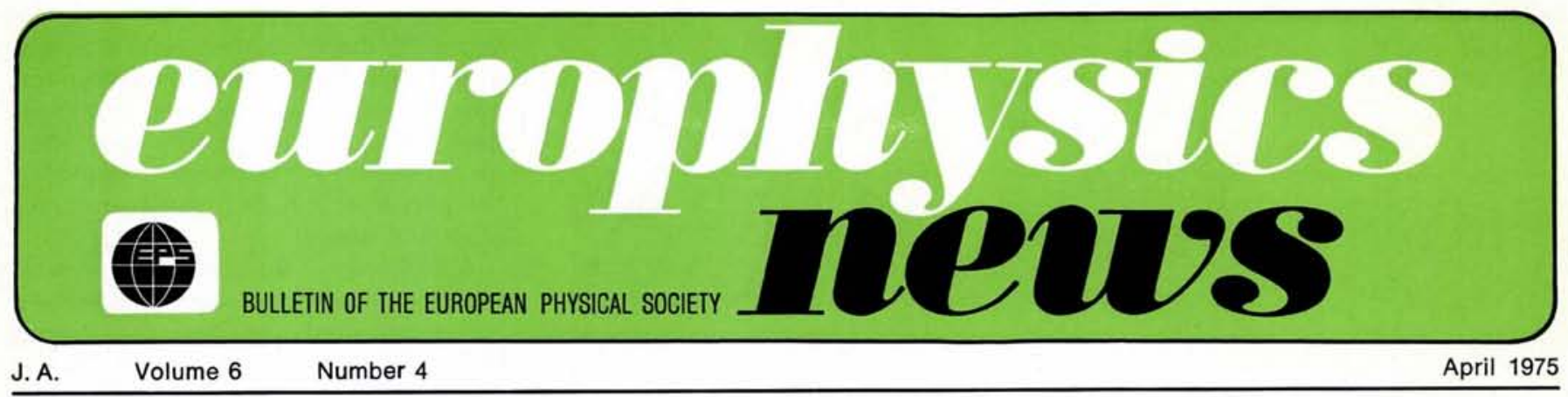

\title{
Radio Telescopes of Large Resolving Power
}

\section{Ryle, Cambridge}

Copyright (C) The Nobel Foundation 1975 Abridged version of the original Nobel Lecture.

It was not until 1958 that it could be shown with some certainty that most of the radio sources were powerful extragalactic objects, but the possibilities were so exciting even in 1952 that my colleagues and I set about the task of designing instruments capable of extending the observation to weaker and weaker sources, and of exploring their internal structure.

Early observations of radio sources were severely limited both by the poor angular resolution and by the limited sensitivity. It was usually impossible to obtain any information about the structure of a source, and adjacent sources could often not be properly separated, whilst attempts to relate the radio sources to optically visible objects were often prevented by the poor positional accuracy. The use of interferometers allowed better positions to be obtained, and sometimes made it possible to derive simple models for the source structure. Few of the sources were found to have an angular size greater than 2-3' arc.

The problem of making detailed maps of such sources arises simply from the fact that the wavelengths used are some million times greater than optical wavelengths - so that even to obtain a radio picture with the same resolution as that of the unaided human eye $\left(\sim 1^{\prime}\right.$ arc) a telescope having a diameter of about $1 \mathrm{~km}$ operating at a wavelength of $50 \mathrm{~cm}$ would be needed. At the same time the instrument would be effective only if the surface accuracy was good enough to make a proper image, corresponding to errors of $\leqslant \lambda / 20$ or a few $\mathrm{cm}$; the engineering problems of building such an instrument are clearly enormous.

An entirely different approach to the problem is to employ small aerial elements which are moved to occupy successively the whole of a much larger aperture plane. The development and use of "aperture synthesis" systems has occupied much of our team in Cambridge over the past 20 years.

The principle of the method is extremely simple. In all methods used to obtain a large resolving power, that is to distinguish the wavefront from a particular direction and ignore those from adjacent directions, we arrange to combine the field measured over as large an area as possible of the wavefront. In a paraboloid we do this by providing a suitably shaped reflecting surface, so that the fields incident on different parts of the sampled wavefront are combined at the focus (Fig. 1a); the voltage produced in the receiving dipole represents the sum of these fields. We can achieve the same result if we use an array of dipoles connected together through equal lengths of cable (Fig. 1b).

Suppose now that only a small part of the wavefront is sampled, but that different parts are sampled in turn (Fig. 1c); could we combine these signals to produce the same effect? Since in general, we do not know the phase of the incident field at different times this would not normally be possible but if we continue to measure one of the samples while we measure the others we can use the signal from this one as a phase reference to correct the values measured in other parts of the wavefront. In this way, by using two small aerial elements, we can again add the fields over the wavefront - the area of which is now determined by the range of relative positions taken by the two aerial elements.

It might be thought that this method would be extremely slow, for if we are

\section{Energy and Physics}

Third General Conference of the European Physical Society

Bucharest, Romania

9-12 September 1975

Participants in the Third General EPS Conference are invited to register at the earliest possible date. Proper accommodation can only be guaranteed if the registration and the fee is received by $\mathbf{3 1}$ July 1975 .

Early registration will also help the organisers of the conference and simplify the customs formalities when entering Romania.

The registration form should be sent to :

A. Corciovei,

Institute for Atomic Physics,

P.O. Box 5206,

Bucharest, Romania.

Eastern participants can pay the registration fee to :

Banque Nationale de Roumanie, Bucharest,

Account No. 64.41.2.01.43.

Western participants can pay

the registration fee to :

Union de Banques Suisses

CH-1211 Geneva 11

Account No. 282.079.30 W

or:

Banque Roumaine

pour le Commerce Extérieur,

Bucharest

Account No. 47.11.032.303-7.

\section{Contents}

Radio Telescopes of Large Resolving Power

The JET Project.

2nd EPS Conference of the Condensed Matter Division on Dielectrics and Phonons

1st EPS Conference of the

Nuclear Physics Division

on Nuclear Interactions at

Medium and Low Energies Society News 


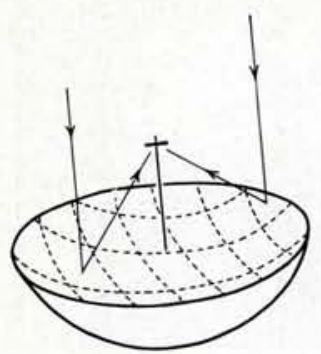

(a)

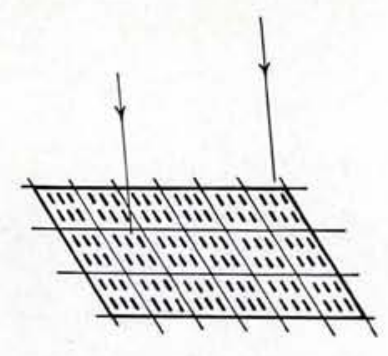

(b)

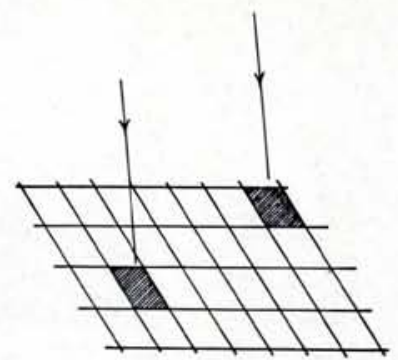

(c)

Fig. 1 The use of (a) a paraboloid, (b) an array of dipoles or (c) the sequential sampling of the wavefront by small aerial elements to achieve a high resolving power by combining the signals from a large part of the incident wavefront.

to sample an area of side D using elements of side $d$, it is necessary to observe with $2 \mathrm{D}^{2} / \mathrm{d}^{2}$ different relative positions of the two aerial elements. In practice, however, the method is not significantly slower than the use of the large equivalent instrument for although a large number of observations must be made, the results may be combined in a computer using additional phase differences, which correspond to many different wave directions (as in a phased array of dipoles), so that with the one set of observations an area of sky may be mapped which is limited only by the diffraction pattern of the small elements themselves; there are in fact some $D^{2} / d^{2}$ different directions which can be scanned in this way, and which would have had to be explored sequentially by a conventional instrument, so that the total observing time of the two methods is nearly the same.

It can also be seen that the sensitivity of the system is much better than would be associated with the small elements, for the signal from a particular point in the sky is contributing to that point on the map for the whole observing period; the resulting signal-to-noise is in fact equivalent to the use of an instrument having a collecting area

$$
2 d^{2}\left(2 D^{2} / d^{2}\right)^{1 / 2} \sim 3 D d,
$$

a figure which may be much greater than that of the elements themselves, and although it is not as great as if the full instrument of area $D^{2}$ had been built, it may exceed that of any instrument which can be built.

Unlike a paraboloid or array, in which both the sensitivity and resolving power are fixed as soon as the wavelength is decided, the value of d may be chosen so that the sensitivity, for any particular wavelength and type of observation, is matched to the resolution.

The method of aperture synthesis avoids the severe structural problems of building very large and accurate paraboloids or arrays, and allows both high resolving power and large effective collecting area to be obtained with a minimum of engineering structure and therefore cost. Provision must be made for the relative movement of the small elements, and their relative positions and electrical connecting paths must be known with an accuracy equal to the surface accuracy of the equivalent instrument $(<\lambda / 20)$. Automatic computing is needed to carry out the Fourier inversion involved in combining the observations to provide a map of the sky.

The first synthesis instruments used a very cheap form of construction; for $\lambda>1 \mathrm{~m}$ an efficient reflecting surface may be provided by thin ( $\sim 1 \mathrm{~mm}$ diameter) wires $5-10 \mathrm{~cm}$ apart. In the case of an instrument operating on $1.7 \mathrm{~m}$, wires stretched across simple parabolic frames of welded steel tube provided a cylindrical paraboloid $450 \mathrm{~m}$ long and $20 \mathrm{~m}$ wide at a cost of about $\$ 5$ per $\mathrm{m}^{2}$.

With the need for still greater resolving power, we realized that physically larger systems operating at metre wavelengths would no longer prove successful, because of the limitation imposed by irregularities of electron density in the ionosphere. But at shorter wavelengths where these are unimportant it becomes difficult to make efficient reflectors by using stretched wires, both because of their deflection by the wind, and because with the closer spacing needed there is difficulty with them twisting together. For operating wavelengths of $<50 \mathrm{~cm}$ a much more rigid supporting structure must be used, and the engineering costs of building a long element become very great.

The obvious solution is to use the system illustrated in Fig. 1c, in which the engineering structure is confined to two small elements - where much higher costs per $\mathrm{m}^{2}$ are acceptable. The method for altering the relative positions of the two elements presents some practical problems; suppose that the elements are mounted on two railway tracks at right angles (Fig. 2b), so that for each position of $A$ on the $\mathrm{N}-\mathrm{S}$ track $\mathrm{B}$ is moved to every position along the E-W track. For values of
$D / d \sim 50$, there are then 5000 different arrangements, and if $\mathrm{B}$ is moved each day, the observations will take 5000 days and although a map will then be available for the whole strip of sky, the period is too long for a graduate student's thesis!

Alternatively B could be moved rapidly - so that several positions could be fitted into the time during which the area of sky remains in the beam of the small elements. This will reduce the total time of the observations, at the expense of observing only parts of the strip of sky. We can clearly extend this period, and so allow more relative positions of $A$ and $B$ each day, if we arrange for the elements to track the chosen point in the sky for an extended period.

As soon as we do this, we realize that the rotation of the earth is itself providing us with a relative motion of $A$ and $B$ as seen from the source, without our having to move them on the surface of the earth at all. Suppose, for example, we have our two elements mounted near the North Pole and we use them to observe an area of sky centred on the Celestial Pole; in this case we do not even have to arrange for them to track. Over a 24 hour period, one will have traced out a circular path about the other (Fig. 3), and the signals recorded during this time can be combined to provide the same response as that of the equivalent ring aerial; by simply altering the separation along a line on say 50 successive days a complete aperture can then be synthesized. Miss Ann Neville and I set up an experimental system in 1960-61 to test the method and develop the computing; we used it to map a region $8^{\circ}$ in diameter round the North Celestial Pole at a wavelength of $1.7 \mathrm{~m}$. We connected up different $14 \mathrm{~m}$ sections of the long cylindrical paraboloid with some other small aerials to stimulate the use of two $14 \mathrm{~m}$ diameter elements at different spacings. The effective diameter of the synthesized instrument was $1 \mathrm{~km}$ and it provided an angular resolution of $4.5^{\prime}$ arc.

As well as showing that the method really worked, it provided some interesting astronomical results - in particular by allowing the detection of sources some eight times weaker

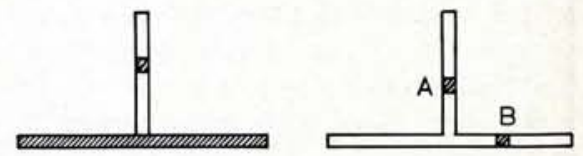

(a)

(b)

Fig. 2 (a) The arrangement used in the instrument built in 1954 by J.H. Blythe.

(b) The equivalent instrument using two small elements. 
than had been observed before; even though the area of sky covered was only some 50 square degrees the results were useful in our cosmological investigations.

In practice only 12 hour observations are needed because of the symmetry of the system and observations need not be made from the North Pole or limited to the Celestial Pole, provided that the elements are situated on an East-West axis, and each is able to track the required region of sky for 12 hours (Fig. $3 b$ ). At low declinations the synthesized instrument becomes elliptical with the north-south aperture reduced by $\sin \delta$. The engineering simplicity of moving the elements along a line, and the consequent great saving in the area of land needed are, however, such great advantages that we eventually built three large instruments in Cambridge with equivalent instrumental diameters of $0.8,1.6$ and nearly $5 \mathrm{~km}$.

These instruments are known as the $1 / 2$-Mile, the 1-Mile and (because its construction coincided with the early negotiations for the entry of Britain into the European Community), the $5 \mathrm{~km}$ Telescopes! The 1 Mile telescope was the first to be built, and this started observations in 1964.

The 1 Mile telescope consists of three $18 \mathrm{~m}$ dishes, two fixed at $0.8 \mathrm{~km}$ spacing, the third mounted on a $0.8 \mathrm{~km}$ rail-track; this arrangement was cheaper than building the longer rail track and it also provided two spacings at a time. It was designed for two main programmes: a) the detection of much fainter and therefore more distant sources in order to explore the early history of the Universe, and so try and distinguish between different cosmological models, and b) to make radio maps of individual sources in an attempt to understand the physical mechanisms within them; most of the sources studied have been powerful extragalactic objects, but the remnants of supernova explosions are perhaps physically as important.

The problem of the physics of radio galaxies and quasars and the cosmological problem are strangely linked; we appear to be living in an evolving Universe, so that very distant sources

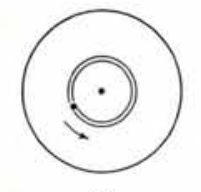

(a) (b)

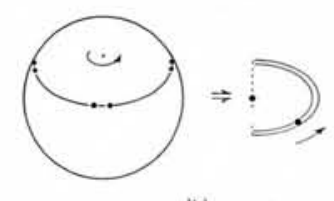

Fig. 3 (a) Two elements mounted near the North Pole observing throughout the day are equivalent to one ring of a much larger instrument.

(b) The elements may be used at other latitudes if arranged on an east-west line and used to track the chosen point for 12 hours. which, due to the signal travel time, we observe as they were when the Universe was younger, may be consistently different from a sample of nearby sources. But the intrinsically most powerful sources are so rare that there are no nearby ones, whilst the weak sources cannot be detected at great distances. If we are to understand how the Universe is evolving, we may first have to solve the physical problem of the individual source - so that we can infer the differences in its evolution at earlier cosmological epochs.

The $1 / 2$-Mile telescope was built later by John Shakeshaft and John Baldwin. It was actually built very cheaply because as can be seen from Fig. 4, it made use of the same rail track, and we were able to get the four $9 \mathrm{~m}$ dishes at scrap-metal prices from a discontinued radio link service, and only the mounts had to be built. It has been used mainly with a radio spectrometer covering the $21 \mathrm{~cm}$ wavelength band of neutral hydrogen to map the distribution of density and velocity of the hydrogen in a number of nearby galaxies, and forms part of a programme concerning the formation and evolution of galaxies.

The $5 \mathrm{~km}$ telescope was completed in 1971, and because it represents a rather more advanced design I will describe it in more detail. It was designed solely for the purpose of mapping individual sources, and besides its larger overall size, the individual dishes are more accurate to allow operation at wavelengths as short as $2 \mathrm{~cm}$. As a result the angular resolution is $\sim 1$ " arc, a figure comparable with the resolution attained by large optical telescopes on good mountain sites. It is at present being used on a wavelength of $6 \mathrm{~cm}$, where the resolution is 2" arc.

In order to improve the speed of observation, four fixed and four movable elements mounted on a rail-track are used, as shown in Fig. 4; this arrangement provides 16 spacings simultaneously, and a single 12 hour observation produces a 2" arc main response with circular grating responses separated by 42 " arc. Sources of smaller extent than 42" arc can therefore be mapped with a single 12 hour observation; more extensive fields of view require further observations with intermediate positions of the movable elements to suppress the grating responses.

For operation at these short wavelengths the positioning of the elements, and the electrical cable connections, must be stable and measured with an accuracy better than $1 \mathrm{~mm}$. Conventional surveying methods allowed each element to be located to

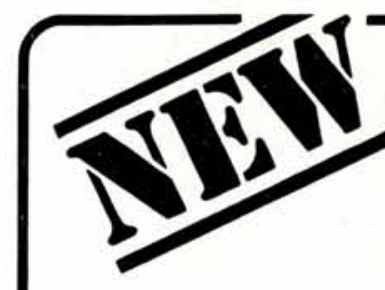

\section{Large Electrostatic}

Accelerators

edited by D. ALLAN BROMLEY 1974. 298 pages. US $\$ 58.50 / \mathrm{Dfl}$. 140.00

In providing a complete discussion of the development, operation, modification and up-grading of all the world's large electrostatic accelerators, this book is without precedent. It represents the vast experience and design expertise of workers in the world's major nuclear science laboratories. Anyone concerned in any way with electrostatic accelerators will find this book an invaluable source of reference.

\section{The Theory of Brillouin Zones and Electronic States in Crystals \\ by $\mathbf{H}$. JONES}

1975. 2nd rev. ed. about 297 pages. US $\$ 37.50 /$ Dfl. 90.00

Written for graduate students possessing the mathematical equipment provided by the average undergraduate course in mathematics or physics, this book treats the theory underlying the classification of electronic states in crystals and the methods by which the eigenvalues of these states may be calculated.

CONTENTS: Introduction. The onedimensional periodic potential. Brillouin zones. The classification of electronic states. Point groups and their representations. Electronic states where the space groups contain glides and screw displacements. Extended k-space. Large zones. Quantitative estimates of wave functions and energies. Spin-orbit coupling effects. Author and Subject Index.

\section{Liquid Phase Epitaxy}

edited by G. M. BLOM, S. L. BLANK and J. M. WOODALL

1974. 342 pages. US $\$ 66.75 / D$ fl. 160.00

The importance of liquid phase epitaxy, particularly in the growth of electronic and magnetic materials for new components of $\mathrm{CW}$ injection lasers, high-efficiency solid state lamps, microwave oscillators and nonvolatile mangnetic bubble memories, is now widely recognised.

This volume brings together not only papers covering liquid phase epitaxial growth but also papers concerned with such material-dependent characteristics as operating life, yield and cost analysis.

North-Holland Publishing Co.

P.O. Box 211

Amsterdam, The Netherlands 


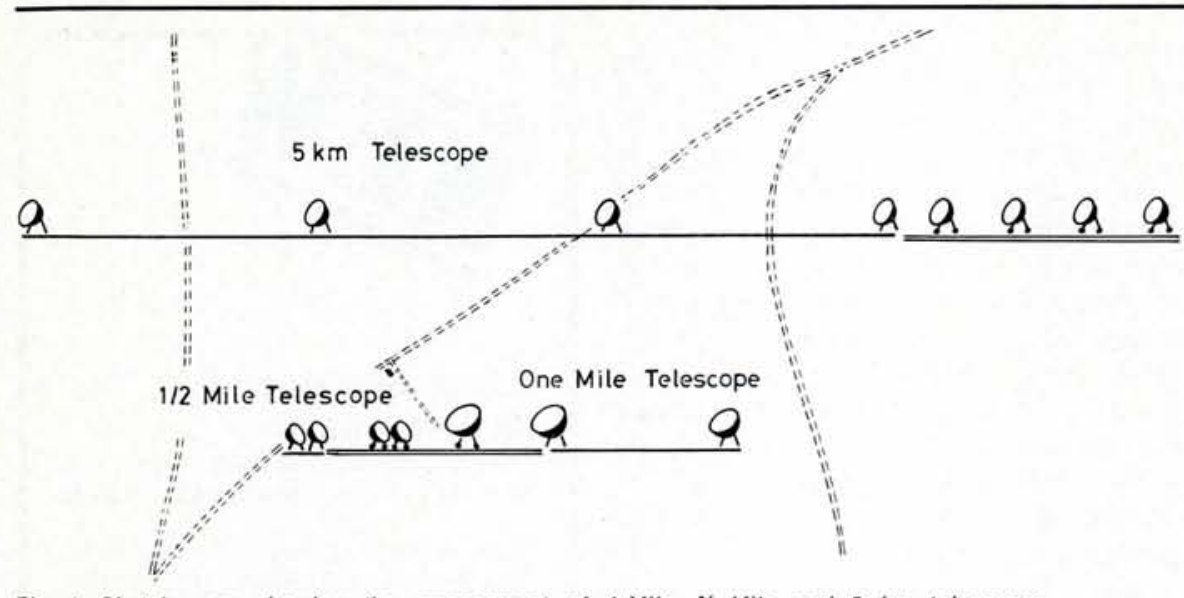

Fig. 4 Sketch map showing the arrangement of 1-Mile, $1 / 2-$ Mile and $5 \mathrm{~km}$ telescopes.

$\pm 10 \mathrm{~mm}$, and the final alignment had to be based entirely on radio observations; the distance between the two outer fixed elements (on which the scale of declination is based) was found in this way to be $3430828 \pm 0.25$ $\mathrm{mm}$, and no changes outside this error have been found over a 2-year period. The combination of azimuth and longitude, on which the measurement of right-ascension depends, was established by observing the bright fundamental star Algol, which is a weak and variable radio source.

The telescope is controlled by an on-line computer which continually up-dates the position of the selected map-centre for precession, aberration etc., and uses this to compute the path differences (corrected for atmospheric refraction) to each pair of elements; these values are then used to control electrical delays in the signals from each element before they are combined in the receivers. The outputs of the receivers are sampled by the computer and stored on a magnetic disc, so that at the completion of the observation they may be combined to form a map of the area observed. The map is then drawn on a curve-plotter controlled by the computer.

This instrument has been used in a wide range of astronomical programmes from the study of ionized hydrogen clouds in our Galaxy to distant quasars. Following the accurate calibration survey it became evident that as an astrometric instrument - to establish a coordinate system across the sky - its measuring accuracy was comparable with the best optical methods, whilst overcoming some of the difficulties in optical work such as the measurement of large angles. Bruce Elsmore is involved in a collaborative programme with optical observers to relate the positions of quasars - (some of which are compact sources at both optical and radio wavelengths) - as measured by radio means, to those derived from the fundamental stars, in order to determine any large-scale non-uniformities which may exist in the present astrometric systems. He also showed how this type of instrument may be used for the direct measurement of astronomical time - without the need for collaborative observations at different longitudes to correct for polar motion - again with an accuracy comparable with optical methods ( $\sim 5 \mathrm{~ms}$ in a 12 hour observation).

Another programme is concerned with a study of the birth of stars; when a cloud of gas condenses to form a star, the dust which it contains provides such an effective screen that newly-formed stars, with their surrounding regions of ionized hydrogen, can never be seen optically; only after this dust cloud has dispersed does the star appear. The dust introduces no appreciable absorption at radio wavelengths, so that radio observations allow these regions to be studied at the earliest stages.

NGC 7538 is an example of such a region, and the upper part of Fig. 5 shows the radio emission as mapped with the 1-Mile telescope. The large diffuse component corresponds almost exactly with the optical nebulosity, and represents the cloud of gas ionized by one or more 0 -stars formed about a million years ago, with the dust sufficiently dispersed to allow the light to be seen. The compact lower component corresponds to gas ionized by much younger stars, which are still embedded in dust too dense for any optical emission to escape, and it is invisible on the photograph. When this southern component was mapped with the higher resolution of the $5 \mathrm{~km}$ telescope, the lower map was obtained, showing that there is an ionized cloud some 10" arc in diameter, probably produced by the radiation from a star of spectral type 08 , and an even more compact cloud to the south of this, produced by a still younger star, only a few thousand years old. The dust surrounding these two compact regions is heated by the young stars they contain, and both have been detected by their infra-red emission.

But the most extensive programme has been the mapping of extragalactic sources - the radio galaxies and quasars; galaxies which, during a brief fraction of their lives, produce some $10^{60}$ ergs of energy, equivalent to the total annihilation of the matter in about a million suns, by a mechanism which is not understood.

Fig. 6 shows the new radio map of the source in the constellation of Cygnus - the first powerful radio galaxy to be recognized. The distribution of polarized emission from the north component is shown in Fig. 7, giving information on the magnetic field.

In most cases the radio emission originates mainly in two huge regions disposed far outside the associated galaxy - although weak emission may also be detectable from a very compact central source coincident with the nucleus of the galaxy. In some cases much more extensive components or a bridge linking the components occur.

The finer detail provided by the $5 \mathrm{~km}$ telescope has already enabled some important conclusions to be drawn; the energy is probably being produced more or less continuously over a period of $10^{7}-10^{8}$ years in a very compact nucleus and not, as was originally thought, in some single explosive event. The source of this energy may be associated with the gravitational collapse of large numbers of stars, in the manner which Tony Hewish described last month, or by material falling into a much more massive collapsed object at the nucleus of the galaxy. The mechanism for transmitting this energy to the compact heads of the main com-

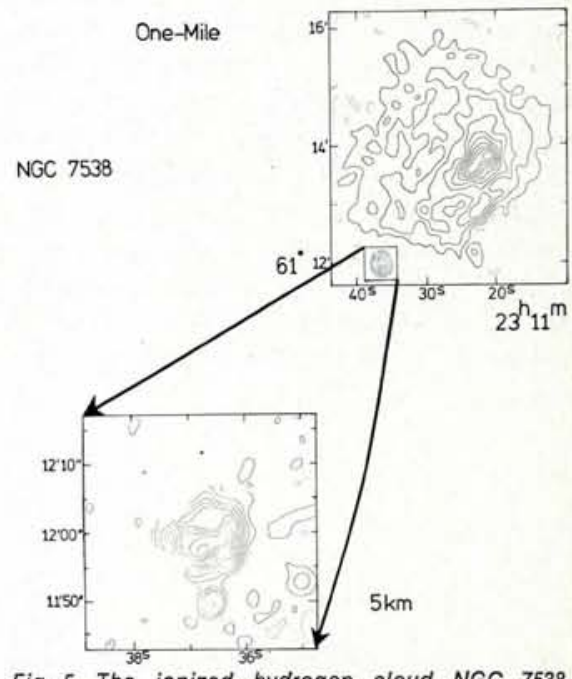

Fig. 5 The ionized hydrogen cloud NGC 7538 The upper map shows the large cloud associated with the optical emission, and another, compact, component to the south. This compact component is shown with greater resolution below. 


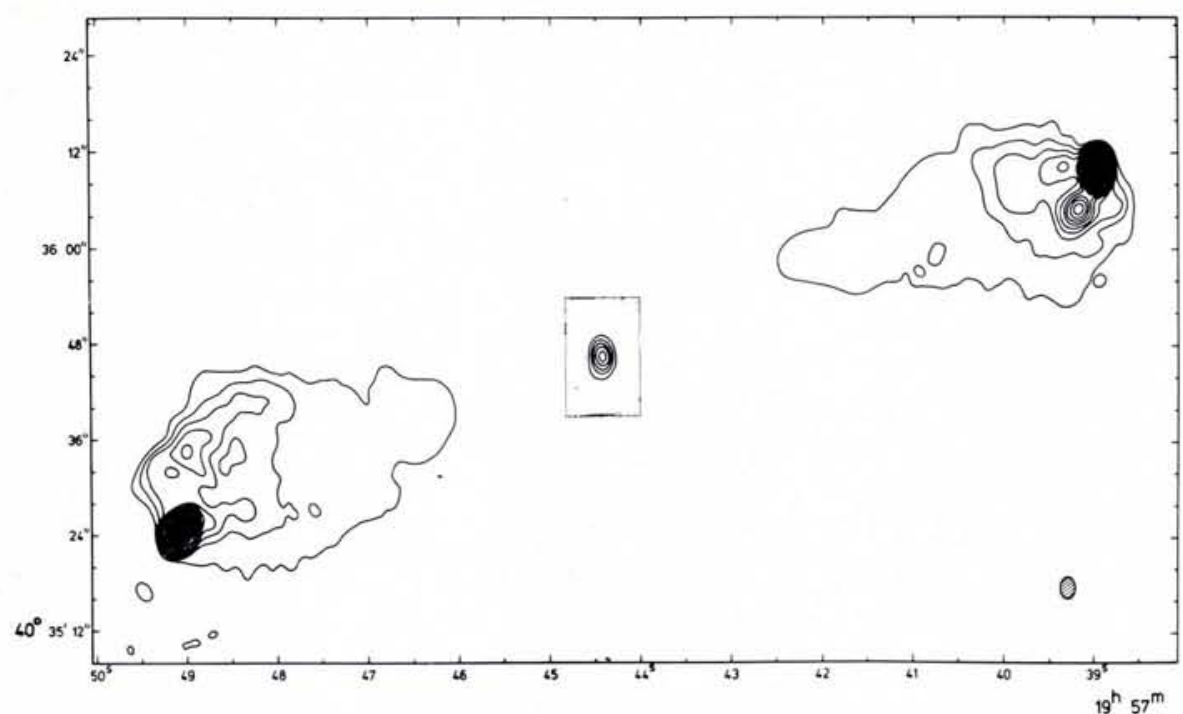

Fig. 6 The powerful radio galaxy, in the constellation of Cygnus mapped with the $5 \mathrm{~km}$ telescope. The compact outer components are exceedingly bright - (31 and 41 contours), The central component - which corresponds to the nucleus of the optical galaxy is very weak and is drawn with contours spaced at $1 / \mathrm{s}$ the interval.

ponents (e.g. Fig. 6) is not under- series of observations with the 1-Mile stood, but may involve a narrow beam of low frequency electromagnetic waves or relativistic particles. The interaction of this beam with the surrounding intergalactic medium might then accelerate the electrons responsible for the radio emission from the compact heads, and their subsequent diffusion into the region behind the heads can probably explain the general shape of the extensive components.

While much remains unanswered, the present conclusions were only reached when detailed maps became available; the physical processes relating the nucleus, the compact heads, and the extensive tails or bridges can clearly only be investigated when the relationship between these structural components is known.

What can we expect in the future? In 1954, the first aperture synthesis telescope provided maps with a resolution of $2.2^{\circ}$; today we have maps with a resolution of 2" arc. Can we foresee a continuing development with radio pictures having much better resolution than the optical ones? The technical problems of increasing the aperture or decreasing the operating wavelength are severe, but they do not appear to be as serious as the limitations imposed by the earth's atmosphere; in optical observations atmospheric turbulence on a scale of $\sim 10 \mathrm{~cm}$ in the lower atmosphere introduces irregularities in the incident wavefront which normally limits the resolution to $\sim 1$ " arc. At radio wavelengths the contribution of these small-scale irregularities is not important, but there are also irregularities of refractive index on a much larger scale in the troposphere. Two distinct types have been found in a observations of a reference point source close to the area to be map- ped, could be made simultaneously at every spacing; the observed phase errors for this reference source could then be used to provide a continuous correction for the signals from the area being mapped.

Such techniques can be extended to interferometers having baselines of many thousands of kilometres (VLBI) but even if adequate phase stability can be attained in this way there are serious practical difficulties in making maps with a resolution $\sim 0.001$ " arc, due to the inevitable poor sampling of the aperture plane. Even with five or six stations distributed across one hemisphere of the world, and using every possible combination of the signals from them, with observing periods lasting several hours, the fraction of the aperture plane which can be filled is still very small, so that the field of view which can be mapped without ambiguity from secondary responses is unlikely to exceed 0.02 " arc. Whilst there seems little hope of deriving complete maps of most sources with this resolution, there are certainly some central components where such a map could provide very important information.

But I think it may also be important for our understanding of the mechanisms operating in the main components of radio sources, to obtain complete maps with intermediate resolution; for this work extensions of the present synthesis techniques, while retaining good filling of the aperture plane, are needed.

I feel very fortunate to have started my research at a time which allowed me and my colleagues to play a part in these exciting developments.

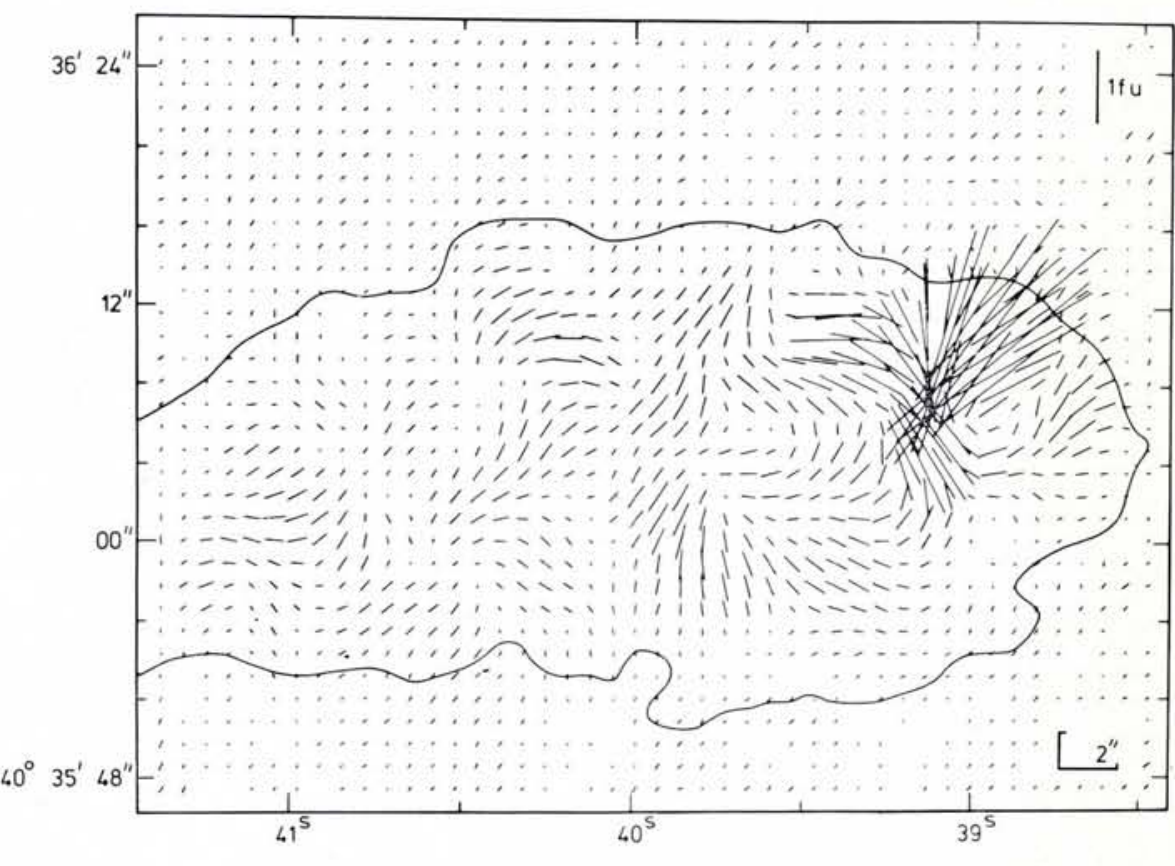

$19^{\mathrm{h}} 57^{\mathrm{m}}$

Fig. 7 The polarization of the emission from the north component of the Cygnus source which shows the magnetic field to be turbulent on a scale $\sim 10^{4}$ light years. 